\title{
The Determinants of the Preferred Walking Speed in Individuals with Obesity
}

\author{
Aitor Fernández Menéndez ${ }^{a} \quad$ Mathieu Saubade $^{b} \quad$ Didier Hans $^{c}$ \\ Grégoire P. Millet ${ }^{a}$ Davide Malatesta ${ }^{a}$ \\ aInstitute of Sport Sciences, Faculty of Biology and Medicine, University of Lausanne, \\ Lausanne, Switzerland; bSports Medicine Unit, Swiss Olympic Medical Center, Lausanne \\ University Hospital, Lausanne, Switzerland; ' ${ }^{c}$ Center for Bone Diseases, Lausanne University \\ Hospital, Lausanne, Switzerland
}

\section{Keywords}

Recovery · Energy cost · Perceived exertion - External work · Self-selected walking speed

\begin{abstract}
Background: The preferred walking speed (PWS), also known as the "spontaneous" or "selfselected" walking speed, is the speed normally used during daily living activities and may represent an appropriate exercise intensity for weight reduction programs aiming to enhance a more negative energy balance. Objectives: The aim of this study was to examine, simultaneously, the energetics, mechanics, and perceived exertion determinants of PWS in individuals with obesity. Methods: Twenty-three adults with obesity (age $32.7 \pm 6.8$ years, body mass index $33.6 \pm 2.6 \mathrm{~kg} / \mathrm{m}^{2}$ ) were recruited. The participants performed 10 min of treadmill familiarization, and PWS was determined. Each subject performed six 5-min walking trials (PWS $0.56,0.83,1.11,1.39$, and $1.67 \mathrm{~m} / \mathrm{s}$ ). Gas exchanges were collected and analyzed to obtain the gross energy cost of walking $\left(\mathrm{G}_{\mathrm{Cw}}\right)$, rated perceived exertion (RPE) was measured using a 6-20 Borg scale, and the external mechanical work $\left(\mathrm{W}_{\text {ext }}\right)$ and the fraction of mechanical energy recovered by the pendular mechanism (Recovery) were computed using an instrumented treadmill. Second-order least-squares regression was used to calculate the optimal walking speed (OWS) of each variable. Results: No significant difference was found between PWS $(1.28 \pm 0.13 \mathrm{~m} / \mathrm{s})$ and $O W S$ for $G_{\mathrm{Cw}}(1.28 \pm 0.10 \mathrm{~m} / \mathrm{s}), R P E$ cost of walking $(1.38 \pm 0.14 \mathrm{~m} / \mathrm{s})$, and Recovery $(1.48 \pm 0.27 \mathrm{~m} / \mathrm{s} ; p>0.06$ for all), but the PWS was significantly faster than the OWS for $W_{\text {ext }}(0.98 \pm 0.56 \mathrm{~m} / \mathrm{s} ; p<0.02)$. Multiple regression $(r=0.72 ; p=0.003)$ showed that $\sim 52 \%$ of the variance in PWS was explained by Recovery, $W_{\text {ext }}$, and height. Conclusion: The main
\end{abstract}


Fernández Menéndez et al.: Optimal and Preferred Walking Speeds in Obesity

finding of this study was that obese adults may select their PWS in function of several competing demands, since this speed simultaneously minimizes pendular energy transduction, energy cost, and perceived exertion during walking. Moreover, recovery of mechanical work, external work, and height seem to be the major determinants of PWS in these individuals.

(C) 2019 The Author(s)

Published by S. Karger AG, Basel

\section{Introduction}

Obesity has been recognized as a chronic progressive disease with a worldwide prevalence that has more than doubled over the past decades, and its prevention and treatment are a public health priority [1]. The weight gain that leads to obesity is the result of an imbalance between energy intake and energy expenditure. Although a decreased level of physical activity [2] may contribute to a reduction in energy expenditure, the role of physical activity in energy balance [3], weight maintenance, and weight loss remains controversial [4-6]. However, higher levels of physical activity are consistently associated with weight loss maintenance [7], and thus may be an active and useful strategy for weight management in obese people $[6,8]$. Walking represents the most common modality of physical activity in daily life and constitutes an important factor of specific nonexercise activity thermogenesis [9]. The preferred walking speed (PWS), also known as the "spontaneous" or "self-selected" walking speed, is the speed normally used during daily living activities as well as an appropriate exercise intensity for weight reduction programs aiming to enhance a more negative energy balance $[10,11]$.

Obese adults prefer to walk at a slower PWS than their lean counterparts [12-15]. This slower preferred pace was commonly associated with several differences in gait pattern, such as a reduction in stride length and frequency, a longer stance and double support phase duration, and a shorter swing phase [12-15]. Moreover, obese individuals walk in a more erect posture, with lower knee and hip flexion as well as higher ankle plantarflexion compared to lean subjects [13]. However, this slower PWS remains very close to the most economical speed (i.e., the speed that corresponds to the lowest energy expenditure per distance [the energy cost of walking]) $[16,17]$. This outcome confirms, in this population, the well-established principle that lean individuals prefer to walk optimizing energy cost [18-20]. Nevertheless, the processes by which people select a PWS close to optimal walking speed (OWS) are not clear, and they emerge from competing demands of several constraints, such as stability, accuracy, and force minimization [20]. Moreover, energy optimization can be modulated by life experiences and people can "learn" how to "tune" the physiology and biomechanics of walking to optimize their gait pattern and to become more economical [20-22]. In fact, as a result of precocious and chronic adaptation to loading, adults with Prader-Willi syndrome, who develop morbid obesity during early childhood, prefer to walk at a slower PWS to improve the pendulum-like mechanism (i.e., pendular energy transduction) compared to adults with nonsyndromal obesity [22]. Therefore, under the chronic extra loading, adults with nonsyndromal obesity can select a slower PWS and can modify their gait pattern to increase the dynamic balance during walking [23], to minimize the load on the knees [13], to reduce the increment of the external mechanical work $\left(W_{\text {ext }}\right)[24]$, and thus to optimize the energy cost of walking at this speed. Moreover, it was previously suggested that the "principle of least effort" may be another important determinant in walking regulation in lean individuals [25]. This author speculated that the central nervous system (CNS) would be able to adapt and control walking based on perceived exertion, readapting the movement patterns to minimize energy expenditure. However, to the best of our knowledge, no studies have been conducted to simultaneously investigate these minimizing strategies during walking in indi- 
Fig. 1. Representative illustration of the assessment of the android/ gynoid fat ratio region.

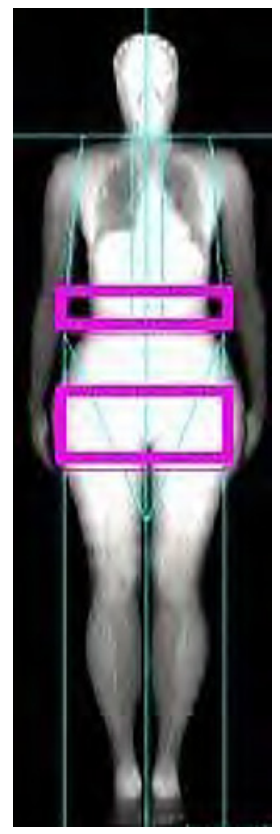

viduals with obesity to better understand how these subjects regulate their gait and select their PWS.

Therefore, the aim of this study was to simultaneously examine the energetics, mechanics, and perceived exertion determinants of the PWS in obese individuals. We hypothesized that individuals with obesity choose a PWS that simultaneously optimizes the energy cost, $\mathrm{W}_{\text {ext, }}$, and perceived exertion of walking.

\section{Methods}

\section{Participants}

Twenty-three obese adults (age 18-45 years, body mass index [BMI] $>30 \mathrm{~kg} / \mathrm{m}^{2}$ ), who were healthy but sedentary ( $\leq 2 \mathrm{~h}$ of physical activity per week over the past year [26]) and free of any neurological, orthopedic, cardiovascular, or respiratory condition, were recruited for this study. They were extensively screened by a physician, who performed a physical examination.

\section{Experimental Design}

The participants were asked to refrain from vigorous physical activity for the $24 \mathrm{~h}$ preceding the experimental session, and they attended the laboratory at least $3 \mathrm{~h}$ following the consumption of their last meal. Prior to testing, anthropometric characteristics and body composition were assessed. Subjects then performed a 10-min treadmill familiarization (T150-FMT-MED; Arsalis, Glabais, Belgium) at the experimental walking speeds [27], and PWS was determined according to the protocol described previously by Martin et al. [28]. After 5 min of resting, each individual completed another 5-min level walking trial at each equally spaced speed $(0.56,0.83,1.11,1.39$, and $1.67 \mathrm{~m} / \mathrm{s})$ in a randomized order on the instrumented treadmill, with 5-min rest periods in between speeds. During the walking trials, metabolic and mechanical data were acquired. 
Fernández Menéndez et al.: Optimal and Preferred Walking Speeds in Obesity

\section{Assessments}

Anthropometric Characteristics. Standing height was measured using a Harpenden stadiometer. Body mass was measured using a precision digital scale with the subject wearing shorts, T-shirt, and shoes.

Body Composition. Dual-energy X-ray absorptiometry (iDXA; GE Healthcare Lunar, WI, USA) was used to assess whole-body composition (lean and fat total body mass, trunk, and upper and lower limbs). The android/gynoid fat ratio was calculated from the regions of interest according to the manufacturer's instructions (Fig. 1). Briefly, the android region of interest included the area between the hip and 20\% of the hip-neck distance. The lateral limits of the android area corresponded to the medial surface of both upper limbs. The gynoid region of interest was placed below the hip, with the upper limit at a distance equal to 1.5 times the height of the android region of interest. The lower limit was set at a distance 3.5 times the height of the android region of interest, and the lateral limits were defined by the medial surface of both lower limbs.

Preferred Walking Speed. The participants started to walk on the treadmill at the lowest experimental speed $(0.56 \mathrm{~m} / \mathrm{s})$, which was gradually increased until the individual subjectively identified the PWS. This procedure was repeated, starting from the highest familiarization speed $(1.67 \mathrm{~m} / \mathrm{s})$ and slowly reducing speed until the PWS was again selected by the subjects. The average of the two speeds selected by the participants (i.e., increasing and decreasing speed trials) was considered the final PWS [29].

Energy Cost of Walking. Prior to the walking trials, expired gases were collected and analyzed during $5 \mathrm{~min}$ in the standing position. Oxygen consumption and $\mathrm{CO}_{2}$ production were continuously measured breath by breath (Oxycon Pro; Jaeger, Höchberg, Germany). Gases and volume calibration were checked before each exercise test. During the standing and walking trials, the metabolic data from the last minute were averaged (steady metabolic state with respiratory exchange ratio $<1$ ) and used to calculate the gross metabolic rate (GMR; W/kg) based on the energy equivalent of oxygen [30]. The GMR was then divided by the corresponding walking speed to obtain the gross energy cost of walking $\left(\mathrm{G}_{\mathrm{Cw}} ; \mathrm{J} / \mathrm{kg} / \mathrm{m}\right)$.

Rated Perceived Exertion. At the end of each walking trial, all subjects were asked for the rated perceived exertion (RPE) using a 6-20 Borg scale [31]. RPE was then divided by the walking speed to calculate the RPE cost of walking ( $\left.\mathrm{RPE}_{\mathrm{Cw}}\right)$.

$W_{\text {ext }}$ and Recovery. The vertical $\left(\mathrm{F}_{\mathrm{v}}\right)$, forward $\left(\mathrm{F}_{\mathrm{f}}\right)$, and lateral $\left(\mathrm{F}_{\mathrm{l}}\right)$ components of the ground reaction forces were acquired from the instrumented treadmill during the last $30 \mathrm{~s}$ of each walking trial at a sampling rate of $1,000 \mathrm{~Hz}$, and 10 consecutive strides were selected [29]. From these data and from the mass of each subject (m), the three-dimensional accelerations of the center of mass were calculated, and, after a mathematical integration, the three components of the velocity changes were obtained $\left(V_{v}, V_{f}\right.$, and $\left.V_{l}\right)$. All strides were selected for analysis when the sum of the increments in $V_{v}, V_{f}$, and $V_{l}$ changes did not differ by more than $25 \%$ from the sum of the decrements [32]. Instantaneous vertical $\left(E_{k v}\right)$, forward $\left(E_{k f}\right)$, and lateral $\left(E_{k l}\right)$ kinetic energies of the center of mass were computed as follows:

$$
E_{k}=E_{k f}+E_{k v}+E_{k l}=0.5 m\left(V_{f}^{2}+V_{v}^{2}+V_{l}^{2}\right)
$$

A second mathematical integration of the vertical component of the velocity $\left(\mathrm{V}_{\mathrm{v}}\right)$ was performed to obtain the height of the center of mass (h). The instantaneous potential energy $\left(E_{p}\right)$ was then computed from $h, m$, and gravity $\left(g=9.81 \mathrm{~m} / \mathrm{s}^{2}\right)$ :

$$
E_{p}=m g h
$$


Table 1. Anthropometric characteristics of the participants

\section{Sex}

Age, years

Height, $m$

Body mass, $\mathrm{kg}$

Body mass index, $\mathrm{kg} / \mathrm{m}^{2}$

Body lean mass, $\mathrm{kg}$

Body fat mass, $\mathrm{kg}$

Trunk lean mass, kg

Trunk fat mass, kg

Trunk fat mass, $\%$

Lower limbs lean mass, $\mathrm{kg}$

Lower limbs fat mass, $\mathrm{kg}$

Lower limbs fat mass, $\%$

Android/gynoid ratio
$19 \mathrm{~F}, 4 \mathrm{M}$

$32.7 \pm 6.8$

$1.70 \pm 0.1$

$97.2 \pm 9.6$

$33.7 \pm 2.6$

$51.8 \pm 8.8$

$42.5 \pm 4.8$

$23.2 \pm 3.8$

$21.8 \pm 3.2$

$47.5 \pm 4.5$

$19.3 \pm 3.4$

$15.4 \pm 3.5$

$43.0 \pm 7.7$

$1.1 \pm 0.2$

Values are mean \pm SD except for sex. F, female; M, male.

The total mechanical energy of the center body mass $\left(E_{t o t}\right)$ was calculated as the sum of the increments in $\mathrm{E}_{\mathrm{k}}$ and $\mathrm{E}_{\mathrm{p}}$ :

$$
E_{t o t}=E_{k}+E_{p}=E_{k f}+E_{k v}+E_{k l}+E_{p}
$$

$\mathrm{W}_{\text {ext }}$ was defined as the sum of the positive increments in $\mathrm{E}_{\text {tot }}$. Throughout this paper, $\mathrm{W}_{\text {ext }}$ is expressed in relative values $(\mathrm{J} / \mathrm{kg} / \mathrm{m})$.

Recovery. In walking, the motion of the center of mass is similar to that of an inverted pendulum [33]. Thus, mechanical energy is recovered within each step by the pendular transduction of $E_{k}$ into $E_{p}$ and vice versa. The fraction of energy recovered due to this transduction was calculated according to previous studies [32]:

$$
\operatorname{Recovery}(\%)=\frac{W_{k}+W_{p}-W_{e x t}}{W_{k}+W_{p}} \times 100
$$

where $W_{k}$ and $W_{p}$ represent the increments of the $\mathrm{E}_{\mathrm{k}}$ and $\mathrm{E}_{\mathrm{p}}$ curves, respectively.

OWS. Second-order least-squares regression was used to model the U-shaped relationship curves between the different variables $\left(\mathrm{G}_{\mathrm{CW}}, \mathrm{RPE}_{\mathrm{Cw}}, \mathrm{W}_{\mathrm{ext}}\right.$ and Recovery) and the walking speed. OWS was then calculated, for each subject and relationship, as the maximal (Recovery) or minimal $\left(\mathrm{G}_{\mathrm{Cw}}, \mathrm{RPE}_{\mathrm{Cw}}\right.$, and $\mathrm{W}_{\text {ext }}$ ) point of the U-shaped curve (i.e., differentiation of each model equation).

\section{Statistical Analysis}

A one-way repeated-measures ANOVA was used to compare the PWS with the OWS for $\mathrm{G}_{\mathrm{Cw}}, \mathrm{RPE}_{\mathrm{Cw}}, \mathrm{W}_{\mathrm{ext}}$, and Recovery. ANOVA was followed by contrasts when the interaction effect was or tended to be significant. Greenhouse-Geisser or Huynh-Feldt adjustments were employed when the assumption of sphericity was violated. Additionally, Bonferroni correction was used to detect significant differences. A paired $t$ test was performed to compare the different variables (GMR, $\mathrm{G}_{\mathrm{Cw}}, \mathrm{RPE}, \mathrm{RPE}_{\mathrm{Cw}}, \mathrm{W}_{\mathrm{ext}}$, and Recovery) assessed at PWS and determined at OWS. Multiple and backward stepwise regression analysis was used to analyze the effects of the anthropometric (age, height, BMI, body mass, total fat, and lean mass), energetic $\left(\mathrm{G}_{\mathrm{CW}}\right)$, and mechanic variables ( $\mathrm{W}_{\mathrm{ext}}$ and Recovery) as well as the RPE on the PWS. Data are expressed as mean \pm standard deviation for all variables, and the level of significance was set at $p<0.05$. 
Fernández Menéndez et al.: Optimal and Preferred Walking Speeds in Obesity

Fig. 2. $\mathrm{W}_{\mathrm{ext}}, \mathrm{G}_{\mathrm{Cw}}, \mathrm{RPE}_{\mathrm{Cw}}$, and Recovery at fixed walking speeds. Circles represent PWS and triangles OWS. * Significant difference with PWS $(p<0.05) .{ }^{\dagger}$ Significant difference with OWS for Recovery $(p<0.05)$. ${ }^{\star}$ Significant difference with OWS for $\mathrm{G}_{\mathrm{Cw}}(p<0.05)$. ${ }^{\S}$ Significant difference with OWS for $\mathrm{RPE}_{\mathrm{Cw}}(p<0.05) . \mathrm{G}_{\mathrm{Cw}}$, gross energy cost of walking; OWS, optimal walking speed; PWS, preferred walking speed; Recovery, fraction of mechanical energy recovered by the pendular mechanism; $\mathrm{RPE}_{\mathrm{Cw}}$, rated perceived exertion cost of walking; $\mathrm{W}_{\text {ext }}$ external mechanical work.

Table 2. Energetics, mechanics, and RPE at PWS and OWS

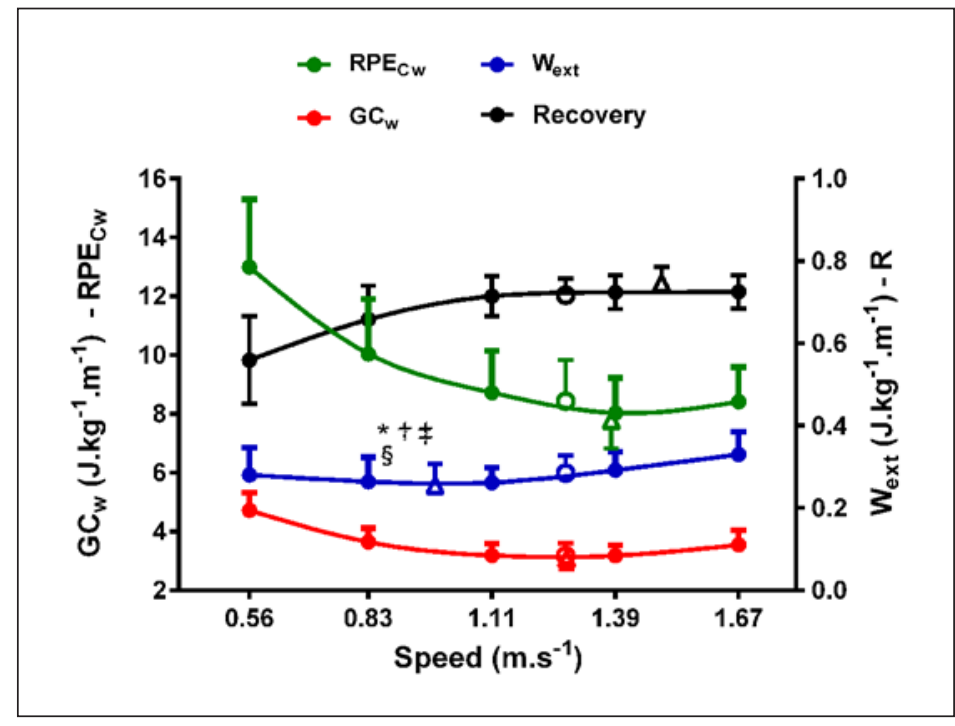

\begin{tabular}{lcc}
\hline & PWS & OWS \\
\hline $\mathrm{G}_{\mathrm{CW}}, \mathrm{J} / \mathrm{kg} / \mathrm{m}$ & $3.2 \pm 0.4$ & $3.1 \pm 0.4^{*}$ \\
$\mathrm{GMR}, \mathrm{W} / \mathrm{kg}$ & $4.0 \pm 0.6$ & $3.9 \pm 0.6$ \\
$\mathrm{Recovery}$ & $0.71 \pm 0.04$ & $0.74 \pm 0.04^{*}$ \\
$\mathrm{~W}_{\text {ext }} \mathrm{J} / \mathrm{kg} / \mathrm{m}$ & $0.29 \pm 0.04$ & $0.26 \pm 0.05^{*}$ \\
$\mathrm{RPE}$ & $10.7 \pm 1.6$ & $10.7 \pm 1.5$ \\
$\mathrm{RPE}_{\mathrm{Cw}}$ & $8.4 \pm 1.4$ & $7.8 \pm 0.9^{*}$ \\
\hline
\end{tabular}

Values are mean $\pm \mathrm{SD}$. $\mathrm{G}_{\mathrm{Cw}}$, gross energy cost of walking; GMR, gross metabolic rate; OWS, optimal walking speed; PWS, preferred walking speed; Recovery, fraction of mechanical energy recovered by the pendular mechanism; RPE, rated perceived exertion; $\mathrm{RPE}_{\mathrm{Cw}}$, rated perceived exertion cost of walking; $\mathrm{W}_{\text {ext }}$ external mechanical work. * Significant difference between PWS and OWS ( $p<0.05)$.

\section{Results}

\section{Participant Characteristics}

The anthropometric characteristics of the participants are reported in Table 1.

\section{PWS versus OWS}

No significant difference was found between PWS $(1.28 \pm 0.13 \mathrm{~m} / \mathrm{s})$ and OWS for $\mathrm{G}_{\mathrm{Cw}}$ $(1.28 \pm 0.10 \mathrm{~m} / \mathrm{s}), \mathrm{RPE}_{\mathrm{Cw}}(1.38 \pm 0.14 \mathrm{~m} / \mathrm{s})$, and Recovery $(1.48 \pm 0.27 \mathrm{~m} / \mathrm{s} ; p>0.06$ for all; Fig. 2), but PWS was significantly faster than OWS for $W_{\text {ext }}(0.98 \pm 0.56 \mathrm{~m} / \mathrm{s} ; p=0.02$; Fig. 2). This OWS for $\mathrm{W}_{\mathrm{ext}}$ was significantly slower than the OWS found for $\mathrm{G}_{\mathrm{CW}}, \mathrm{RPE}_{\mathrm{CW}}$, and Recovery $(p<0.01$ for all; Fig. 2).

Recovery was slightly but significantly lower at PWS than at the optimal speed for this variable $(-4 \% ; p<0.001$; Table 2$)$. At $\mathrm{PWS}, \mathrm{G}_{\mathrm{CW}}, \mathrm{RPE}_{\mathrm{Cw}}$, and $\mathrm{W}_{\text {ext }}$ were significantly higher than these values at their respective OWS $(+3,+8$, and $11 \% ; p=0.008, p=0.007$, and $p=0.005$, respectively; Table 2). However, RPE and GMR at PWS were not significantly different than at OWS ( $p>0.35$ for both; Table 2). A significant correlation was found between PWS and $\operatorname{RPE}_{\mathrm{CW}}(r=-0.43 ; p=0.04)$. 
Fig. 3. Representative illustration of the determinants of PWS in individual with obesity. PWS, preferred walking speed.

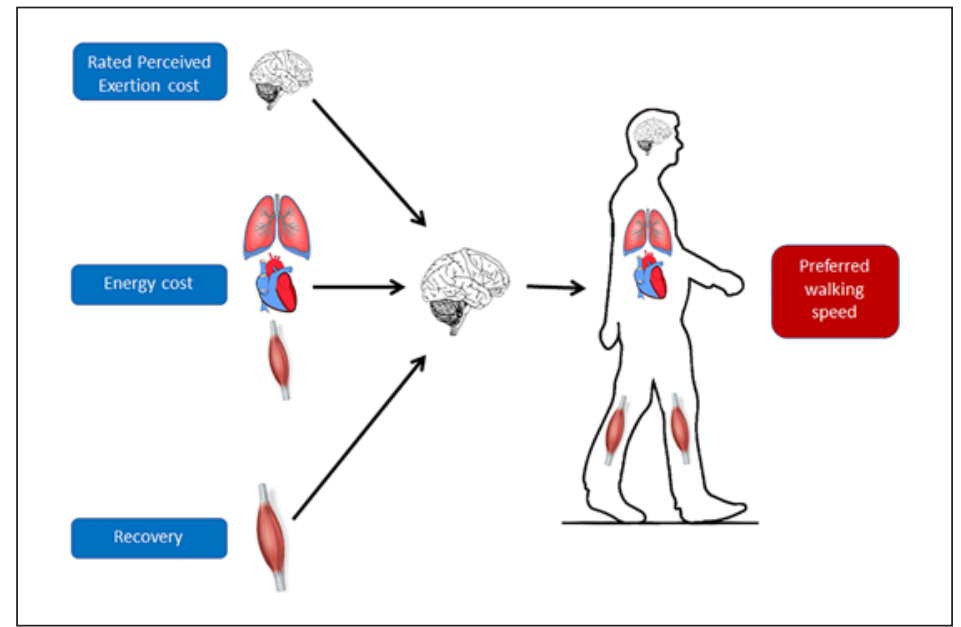

Multiple regression analysis $\left(r=0.72 ; r^{2}=0.52\right.$; adjusted $r^{2}=0.45$; standard error of estimate $= \pm 0.09 ; p=0.003$ ) showed that $\sim 52 \%$ of the variance in PWS was explained by Recovery (standardized coefficient $=0.7 ; p=0.02$ ), $\mathrm{W}_{\text {ext }}$ (standardized coefficient $=1 ; p<$ 0.001 ), and height (standardized coefficient $=0.36 ; p=0.04$ ).

\section{Discussion}

The main finding of this study was that obese adults may select their PWS in function of several competing demands since this speed simultaneously minimizes pendular energy transduction, energy cost, and perceived exertion during walking (Fig. 3). Moreover, Recovery, external work, and height seem to be the major determinants of PWS in these individuals.

The PWS selected by our group in this study $(1.28 \pm 0.13 \mathrm{~m} / \mathrm{s})$ was in agreement with previous studies that reported similar values for this population [14, 34, 35]. However, Browning and Kram [17] measured a higher PWS (1.40 m/s) in adults with the same level of obesity (class I). The slower PWS that we found might be due to methodological differences [36]. In fact, these authors measured the PWS outdoors, whereas in our study, a treadmill was used. Our PWS values are in line with several articles that reported a slower self-selected speed in obese adults than in normal body weight individuals [11, 14, 15, 37, 38]. This slower PWS is usually associated with a shorter step length and a lower step frequency, increasing the time spent during the double support as well as walking with wider steps in obese adults [13-16]. Some authors suggested that these self-selected speeds may be inversely correlated with BMI $[16,17]$, and these gait modifications may arise from anthropometric characteristics such as thigh diameter. Although no correlation was found between PWS and BMI and body composition parameters, multiple regression showed that part of the PWS variance was explained by height. This result confirms previous findings that demonstrated that height explained 30\% of the variation in walking speed [39]. Moreover, Błaszczyk et al. [40] reported a correlation between body height and limb length with PWS in obese adults, suggesting that these anthropometric characteristics may substantially affect self-selected speed.

Other authors hypothesized that the selection of PWS is aimed at optimizing the gait energy cost [41]. This hypothesis was also confirmed by our results, with no difference between PWS and $\mathrm{OWS}$ for $\mathrm{G}_{\mathrm{CW}}$, corroborating previous findings that suggested that the self-selected speed is very close to the walking speed that minimizes energy expenditure (Fig. 2; Table 2) $[18,19]$. However, other factors such as pain [16], impaired balance [11], or $\mathrm{W}_{\text {ext }}[15,42]$ may affect the 
Fernández Menéndez et al.: Optimal and Preferred Walking Speeds in Obesity

selection of PWS in individuals with higher levels of adiposity. In fact, Malatesta et al. [15] suggested that obese individuals chose a slower PWS to minimize $W_{\text {ext }}$ during walking. However, the PWS found in our study was significantly faster than the OWS for $\mathrm{W}_{\text {ext }}(+24 \%$; Fig. 2), with higher $W_{\text {ext }}$ at $P W S$ than at OWS (+11\%; Table 2). In contrast, there was no significant difference between PWS and OWS for Recovery (Fig. 2), with Recovery values at PWS that were slightly lower $\left(-4 \%\right.$; Table 2). The OWS for $\mathrm{W}_{\text {ext }}(0.98 \pm 0.56 \mathrm{~m} / \mathrm{s})$ found in our group of obese individuals is slower than previously reported optimal speed values for lean individuals $(1.1 \mathrm{~m} / \mathrm{s})$ $[43,44]$, whereas the OWS for Recovery $(1.48 \pm 0.27 \mathrm{~m} / \mathrm{s})$ found in our study is similar to the optimal speed previously reported in normal body weight individuals [43, 44]. Although our findings showed these significant differences in terms of $W_{\text {ext }}$ between $P W S$ and OWS, regression analysis revealed that $\sim 52 \%$ of the PWS variation was explained by Recovery, $\mathrm{W}_{\text {ext }}$ and height (i.e., the main determinants of PWS). The latter analysis highlights that, due to the excess of body mass, obese individuals might select a PWS that reduces the increased muscular effort and work to translate the center of mass with respect to the ground (i.e., $\mathrm{W}_{\text {ext }}$ ) [15] and to improve pendular energy transduction (i.e., Recovery) similar to adults with Prader-Willi syndrome [22] and African women carrying loads on their heads [44]. This optimization of pendular energy exchange would be an adaptive mechanism against the extra load to minimize $\mathrm{W}_{\text {ext }}$ and energy cost of walking [22, 43,45]. In fact, although some of the mechanical and energetic variables at PWS and OWS are slightly but significantly different (Fig. 2; Table 2), the values of these variables of PWS were close to those of OWS and were in the region where the relationships were relatively flat (i.e., the bottom/top of the U-shaped curves; Fig. 2) [16]. Taken together, these findings suggest that individuals with obesity may select a PWS in terms of the function of several factors, such as external work, pendular transduction, and energy cost of walking. According to this, some authors suggested that human locomotion is affected by multifactorial interactions resulting from both neural and mechanical aspects [46, 47]. Donelan et al. [48] found that the CNS is able to adjust the gait pattern based on sensory receptors to control stability with a metabolic cost. However, Wong et al. [49] reported that blood gas receptors are not used to optimize movements to minimize energy cost and that the CNS may estimate energy cost from other sensory signals. For instance, it has also been suggested that, in lean individuals, the CNS objective during locomotion is the minimization of perceived exertion (i.e., the "principle of least effort") [25]. This approach is in line with our results, with a correlation found between PWS and $\mathrm{RPE}_{\mathrm{Cw}}$, along with no difference between PWS and OWS for $\mathrm{RPE}_{\mathrm{CW}}$ (Fig. 2) and similar values of RPE at PWS and its OWS (Table 2). This is the first study to experimentally corroborate the suggestions of Marcora [25] in obese individuals, for whom exercise tolerance is a more limiting factor than got lean individuals. Moreover, our findings confirm previous results which showed that the step frequency chosen corresponds to the one perceived as the least strenuous in different conditions [20], and they suggest that, compared with lean individuals, the slower PWS selected in obese adults may also be explained by their higher perception of effort in a physical task [10]. Future studies should further investigate other potential factors that may explain additional variance in the selection of PWS in obese individuals, such as affective experiences [50] and genetic factors [51].

Some limitations need to be addressed. First, the inclusion of a control group with lean individuals may provide a way to show the differences in the selection of PWS between obese and lean individuals. However, several studies have already reported that PWS is slower in obese than in lean individuals [12-15] and inversely correlated with body mass and BMI [15]. Moreover, several studies have been already been conducted to investigated the factors affecting the selection of PWS in lean individuals [18-20]. For these reasons and for clarity's sake, we chose to use only one group of obese individuals to focus our study only on the specific determinants of PWS in this population. Second, the participants of our study belonged to either class I or II of obesity. Therefore, a more marked difference between the PWS selected 
Fernández Menéndez et al.: Optimal and Preferred Walking Speeds in Obesity

and OWS may exist in a group of adults with obesity class III with a slower PWS. In fact, a PWS of only $0.75 \mathrm{~m} / \mathrm{s}$ in a group of women with obesity class III [52] and of $1.09 \mathrm{~m} / \mathrm{s}$ for a group of men with the same class of obesity [14] have been reported. Third, the use of a treadmill may lead to an underestimation of PWS as well as an overestimation of oxygen cost [53]. In fact, two studies reported a slower PWS on the treadmill compared to over ground in young and older individuals related to "safety-related" gait adaptions, such as reduced step length, increased step frequency, and double support duration, which are also distinctive features of obese adults [36,53]. Fourth, the internal mechanical work (i.e., the energy fluctuations of the segments with respect to the center of the body mass) was not assessed in this study. This may contribute to determine the role of mechanical work on PWS [54]. However, the calculation of the internal mechanical work requires several assumptions about segment properties and transfer of energy between them, which may lead to an uncertainty in the calculation of the mechanical work, especially in obese adults [44].

In conclusion, obese adults select a PWS from competing demands of several constraints. This speed minimizes, simultaneously, pendular energy transduction, energy, and effort perceived costs during walking (Fig. 3). Furthermore, Recovery, $\mathrm{W}_{\text {ext }}$ and height seem to be the main determinants of PWS in these individuals. This outcome may be due to the higher body mass in these subjects, which could lead to greater local muscle effort and external work performance, highlighting the importance of inducing a loss in body mass for decreasing $\mathrm{W}_{\text {ext }}$ and subsequently for increasing PWS in this population.

\section{Statement of Ethics}

All subjects gave written informed consent prior to testing. The study was approved by the local ethics committee (Cantonal Swiss Ethics Committees on research involving humans; CER-VD).

\section{Disclosure Statement}

The authors have no conflicts of interest to declare. This research did not receive any specific grant from funding agencies in the public, commercial, or not-for-profit sectors.

\section{References}

1 Collaboration NC; NCD Risk Factor Collaboration (NCD-RisC). Trends in adult body-mass index in 200 countries from 1975 to 2014: a pooled analysis of 1698 population-based measurement studies with $19 \cdot 2$ million participants. Lancet. 2016 Apr;387(10026):1377-96.

2 Guthold R, Stevens GA, Riley LM, Bull FC. Worldwide trends in insufficient physical activity from 2001 to 2016 : a pooled analysis of 358 population-based surveys with 1.9 million participants. Lancet Glob Health. 2018 Oct; 6(10):e1077-86.

3 Blundell JE, Gibbons C, Caudwell P, Finlayson G, Hopkins M. Appetite control and energy balance: impact of exercise. Obes Rev. 2015 Feb;16 Suppl 1:67-76.

4 Pontzer H, Durazo-Arvizu R, Dugas LR, Plange-Rhule J, Bovet P, Forrester TE, et al. Constrained Total Energy Expenditure and Metabolic Adaptation to Physical Activity in Adult Humans. Curr Biol. 2016 Feb;26(3):410-7.

5 Jakicic JM, Rogers RJ, Davis KK, Collins KA. Role of Physical Activity and Exercise in Treating Patients with Overweight and Obesity. Clin Chem. 2018 Jan;64(1):99-107.

6 Jakicic JM, Marcus BH, Gallagher KI, Napolitano M, Lang W. Effect of exercise duration and intensity on weight loss in overweight, sedentary women: a randomized trial. JAMA. 2003 Sep;290(10):1323-30.

7 Ostendorf DM, Caldwell AE, Creasy SA, Pan Z, Lyden K, Bergouignan A, et al. Physical Activity Energy Expenditure and Total Daily Energy Expenditure in Successful Weight Loss Maintainers. Obesity (Silver Spring). 2019 Mar;27(3):496-504. 
Fernández Menéndez et al.: Optimal and Preferred Walking Speeds in Obesity

8 Hill JO, Peters JC, Wyatt HR. Using the energy gap to address obesity: a commentary. J Am Diet Assoc. 2009 Nov; 109(11):1848-53.

9 Levine JA, McCrady SK, Lanningham-Foster LM, Kane PH, Foster RC, Manohar CU. The role of free-living daily walking in human weight gain and obesity. Diabetes. 2008 Mar;57(3):548-54.

10 Hills AP, Byrne NM, Wearing S, Armstrong T. Validation of the intensity of walking for pleasure in obese adults. Prev Med. 2006 Jan;42(1):47-50.

11 Browning RC. Locomotion mechanics in obese adults and children. Curr Obes Rep. 2012;1(3):152-9.

12 de Souza SA, Faintuch J, Valezi AC, Sant' Anna AF, Gama-Rodrigues JJ, de Batista Fonseca IC, et al. Gait cinematic analysis in morbidly obese patients. Obes Surg. 2005 Oct;15(9):1238-42.

13 DeVita P, Hortobágyi T. Obesity is not associated with increased knee joint torque and power during level walking. J Biomech. 2003 Sep;36(9):1355-62.

14 Spyropoulos P, Pisciotta JC, Pavlou KN, Cairns MA, Simon SR. Biomechanical gait analysis in obese men. Arch Phys Med Rehabil. 1991 Dec;72(13):1065-70.

15 Malatesta D, Vismara L, Menegoni F, Galli M, Romei M, Capodaglio P. Mechanical external work and recovery at preferred walking speed in obese subjects. Med Sci Sports Exerc. 2009 Feb;41(2):426-34.

16 Browning RC, Baker EA, Herron JA, Kram R. Effects of obesity and sex on the energetic cost and preferred speed of walking. J Appl Physiol (1985). 2006 Feb;100(2):390-8.

17 Browning RC, Kram R. Energetic cost and preferred speed of walking in obese vs. normal weight women. Obes Res. 2005 May;13(5):891-9.

18 Cavagna GA, Kaneko M. Mechanical work and efficiency in level walking and running. J Physiol. 1977 Jun; 268(2):467-81.

19 Rose J. JG G: Human Walking. 3rd ed. Philadelphia (PA): Lippincott Williams \& Wilkins; 2006. p. 273.

20 Selinger JC, O'Connor SM, Wong JD, Donelan JM. Humans Can Continuously Optimize Energetic Cost during Walking. Curr Biol. 2015 Sep;25(18):2452-6.

21 Ivanenko YP, Dominici N, Lacquaniti F. Development of independent walking in toddlers. Exerc Sport Sci Rev. 2007 Apr;35(2):67-73.

22 Malatesta D, Vismara L, Menegoni F, Grugni G, Capodaglio P. Effect of obesity onset on pendular energy transduction at spontaneous walking speed: Prader-Willi versus nonsyndromal obese individuals. Obesity (Silver Spring). 2013 Dec;21(12):E586-91.

23 Hills AP, Hennig EM, Byrne NM, Steele JR. The biomechanics of adiposity - structural and functional limitations of obesity and implications for movement. Obes Rev. 2002 Feb;3(1):35-43.

24 Cavagna GA, Saibene FP, Margaria R. External work in walking. J Appl Physiol. 1963 Jan;18(1):1-9.

25 Marcora SM. The variable monitored by the CNS to continuously optimize walking: energy cost or perception of effort? Curr Biol. 2015. https://www.researchgate.net/publication/282757756_The_variable_monitored_ by_the_CNS_to_continuously_optimize_walking_energy_cost_or_perception_of_effort.

26 Haskell WL, Lee IM, Pate RR, Powell KE, Blair SN, Franklin BA, et al. Physical activity and public health: updated recommendation for adults from the American College of Sports Medicine and the American Heart Association. Med Sci Sports Exerc. 2007 Aug;39(8):1423-34.

27 Wall JC, Charteris J. A kinematic study of long-term habituation to treadmill walking. Ergonomics. 1981 Jul; 24(7):531-42.

28 Martin PE, Rothstein DE, Larish DD. Effects of age and physical activity status on the speed-aerobic demand relationship of walking. J Appl Physiol (1985). 1992 Jul;73(1):200-6.

29 Fernández Menéndez A, Saudan G, Sperisen L, Hans D, Saubade M, Millet GP, et al. Effects of Short-Term Normobaric Hypoxic Walking Training on Energetics and Mechanics of Gait in Adults with Obesity. Obesity (Silver Spring). 2018 May;26(5):819-27.

30 Astrand PO. Textbook of work physiology. New York: McGraw-Hill Series in Health Ed.; 1986.

31 Borg GA. Psychophysical bases of perceived exertion. Med Sci Sports Exerc. 1982;14(5):377-81.

32 Massaad F, Lejeune TM, Detrembleur C. The up and down bobbing of human walking: a compromise between muscle work and efficiency. J Physiol. 2007 Jul;582(Pt 2):789-99.

33 Cavagna GA, Thys H, Zamboni A. The sources of external work in level walking and running. J Physiol. 1976 Nov;262(3):639-57.

34 Lai PP, Leung AK, Li AN, Zhang M. Three-dimensional gait analysis of obese adults. Clin Biomech (Bristol, Avon). 2008;23 Suppl 1:S2-6.

35 Mattsson E, Larsson UE, Rössner S. Is walking for exercise too exhausting for obese women? Int J Obes Relat Metab Disord. 1997 May;21(5):380-6.

36 Malatesta D, Canepa M, Menendez Fernandez A. The effect of treadmill and overground walking on preferred walking speed and gait kinematics in healthy, physically active older adults. Eur J Appl Physiol. 2017 Sep; 117(9):1833-43.

37 Dufek JS, Currie RL, Gouws PL, Candela L, Gutierrez AP, Mercer JA, et al. Effects of overweight and obesity on walking characteristics in adolescents. Hum Mov Sci. 2012 Aug;31(4):897-906.

38 McGraw B, McClenaghan BA, Williams HG, Dickerson J, Ward DS. Gait and postural stability in obese and nonobese prepubertal boys. Arch Phys Med Rehabil. 2000 Apr;81(4):484-9.

39 Samson MM, Crowe A, de Vreede PL, Dessens JA, Duursma SA, Verhaar HJ. Differences in gait parameters at a preferred walking speed in healthy subjects due to age, height and body weight. Aging (Milano). 2001 Feb; 13(1):16-21. 
Fernández Menéndez et al.: Optimal and Preferred Walking Speeds in Obesity

40 Błaszczyk JW, Plewa M, Cieślinska-Swider J, Bacik B, Zahorska-Markiewicz B, Markiewicz A. Impact of excess body weight on walking at the preferred speed. Acta Neurobiol Exp (Wars). 2011;71(4):528-40.

41 Bertram JE, Ruina A. Multiple walking speed-frequency relations are predicted by constrained optimization. J Theor Biol. 2001 Apr;209(4):445-53.

42 Browning RC, McGowan CP, Kram R. Obesity does not increase external mechanical work per kilogram body mass during walking. J Biomech. 2009 0ct;42(14):2273-8.

43 Cavagna GA, Willems PA, Legramandi MA, Heglund NC. Pendular energy transduction within the step in human walking. J Exp Biol. 2002 Nov;205(Pt 21):3413-22.

44 Willems PA, Cavagna GA, Heglund NC. External, internal and total work in human locomotion. J Exp Biol. 1995 Feb;198(Pt 2):379-93.

45 Heglund NC, Willems PA, Penta M, Cavagna GA. Energy-saving gait mechanics with head-supported loads. Nature. 1995 May;375(6526):52-4.

46 Sousa AS, Silva A, Tavares JM. Biomechanical and neurophysiological mechanisms related to postural control and efficiency of movement: a review. Somatosens Mot Res. 2012;29(4):131-43.

47 O'Connor SM, Donelan JM. Fast visual prediction and slow optimization of preferred walking speed. J Neurophysiol. 2012 May;107(9):2549-59.

48 Donelan JM, Shipman DW, Kram R, Kuo AD. Mechanical and metabolic requirements for active lateral stabilization in human walking. J Biomech. 2004 Jun;37(6):827-35.

49 Wong JD, O'Connor SM, Selinger JC, Donelan JM. Contribution of blood oxygen and carbon dioxide sensing to the energetic optimization of human walking. J Neurophysiol. 2017 Aug;118(2):1425-33.

50 Ekkekakis P. Let them roam free? Physiological and psychological evidence for the potential of self-selected exercise intensity in public health. Sports Med. 2009;39(10):857-88.

51 den Hoed M, Brage S, Zhao JH, Westgate K, Nessa A, Ekelund U, et al. Heritability of objectively assessed daily physical activity and sedentary behavior. Am J Clin Nutr. 2013 Nov;98(5):1317-25.

52 Ohrström M, Hedenbro J, Ekelund M. Energy expenditure during treadmill walking before and after vertical banded gastroplasty: a one-year follow-up study in 11 obese women. Eur J Surg. 2001 Nov;167(11):845-50.

53 Dal U, Erdogan T, Resitoglu B, Beydagi H. Determination of preferred walking speed on treadmill may lead to high oxygen cost on treadmill walking. Gait Posture. 2010 Mar;31(3):366-9.

54 Peyré-Tartaruga LA, Coertjens M. Locomotion as a powerful model to study integrative physiology: efficiency, economy, and power relationship. Front Physiol. 2018 Dec;9:1789. 